\title{
Classification of unlabeled observations in Species Distribution Modelling using Point Process Models.
}

\author{
Emy Guilbault ${ }^{1}$, Ian Renner ${ }^{1}$, Michael Mahony ${ }^{1}$, and Eric Beh ${ }^{1}$ \\ ${ }^{1}$ The University of Newcastle Faculty of Science
}

October 23, 2020

\begin{abstract}
1. Species distribution modelling, which allows users to predict the spatial distribution of species with the use of environmental covariates, has become increasingly popular, with many software platforms providing tools to fit species distribution models. However, the species observations used in species distribution models can have varying levels of quality and can have incomplete information, such as uncertain species identity. 2. In this paper, we develop two algorithms to reclassify observations with unknown species identities which simultaneously predict different species distributions using spatial point processes. We compare the performance of the different algorithms using different initializations and parameters with models fitted using only the observations with known species identity through simulations. 3. We show that performance varies with differences in correlation among species distributions, species abundance, and the proportion of observations with unknown species identities. Additionally, some of the methods developed here outperformed the models that didn't use the misspecified data. 4. These models represent an helpful and promising tool for opportunistic surveys where misidentification happens or for the distribution of species newly separated in their taxonomy.
\end{abstract}

\section{Hosted file}

Article_main.pdf available at https://authorea.com/users/369598/articles/488425classification-of-unlabeled-observations-in-species-distribution-modelling-using-pointprocess-models 